\title{
Knockdown of Aurora-B inhibits the growth of non-small cell lung cancer A549 cells
}

\author{
JING JING YU ${ }^{1 *}$, LONG DIAN ZHOU ${ }^{2 *}$, TIAN TIAN ZHAO ${ }^{1}$, WEI BAI ${ }^{1}$, JING ZHOU $^{1}$ and WEI ZHANG ${ }^{1}$ \\ Departments of ${ }^{1}$ Respiratory Medicine and ${ }^{2}$ Orthopedics, The First Affiliated Hospital of Nanchang University, \\ Nanchang, Jiangxi 330006, P.R. China
}

Received August 15, 2014; Accepted May 20, 2015

DOI: $10.3892 / \mathrm{ol} .2015 .3467$

\begin{abstract}
Elevated expression of Aurora-B affects cell apoptosis and proliferation in a variety of solid tumors. However, the role of Aurora-B has been poorly evaluated in non-small cell lung cancer (NSCLC). In the present study, it was found that Aurora-B was overexpressed in tissue specimens obtained from 174 patients with lung cancer. It was also demonstrated that knockdown of Aurora-B induces apoptosis and inhibits the growth of lung cancer A549 cells in vitro and in vivo. Furthermore, it was found that silencing Aurora-B decreased the activity of the phosphoinositide 3-kinase (PI3K)/AKT pathway. Therefore, it was concluded that knockdown of Aurora-B induces apoptosis and inhibits growth in NSCLC A549 cells, in addition to inhibiting the activity of the PI3K/AKT signaling pathway. Targeting Aurora-B may provide a novel target for lung cancer therapy.
\end{abstract}

\section{Introduction}

Lung cancer is the leading cause of cancer-associated mortality worldwide. Non-small cell lung carcinoma (NSCLC) accounts for $\sim 80 \%$ of lung tumors. Although chemotherapy has been increasingly used to treat patients with NSCLC, the five-year survival rate of patients with NSCLC is $<15 \%$ and has not improved over the previous decades (1). Therefore, the development of more widely effective targeted agents for NSCLC treatment is required.

The Aurora kinases play a critical role in mitosis, and the kinases have been indicated to be promising targets for cancer therapy due to their frequent overexpression in a variety of

Correspondence to: Professor Wei Zhang, Department of Respiratory Medicine, The First Affiliated Hospital of Nanchang University, 17 Yong Wai Zheng, Nanchang, Jiangxi 330006, P.R. China

E-mail: zhangwei8869@126.com

*Contributed equally

Key words: non-small cell lung cancer, Aurora-B, apoptosis, growth, PI3K/AKT signaling pathway tumors (2-4). Aurora-B is one of the major protein kinases that ensures the correct execution and fidelity of mitosis. The increased expression of Aurora-B is evident in various cancers, and its upregulated expression has been identified as associated with clinical aggressiveness (5-8). Li et al revealed that the downregulation of Aurora-B inhibits proliferation and metastasis, induces $\mathrm{G} 2 / \mathrm{M}$ phase arrest in clear cell renal cell carcinoma cells and exerts antitumor activity in a SN12C xenograft model (9). These findings indicated that Aurora-B possesses oncogenic characteristics and plays a key role in the malignant behavior of tumors, suggesting that Aurora-B is an important anti-tumor target. However, there is a lack of data for the association between the elevated expression of Aurora-B and the malignant potential of NSCLC lesions.

In the present study, immunohistochemistry (IHC) was performed to investigate the expression levels of the Aurora-B protein in 174 NSCLC tissues and 174 adjacent tissues. Furthermore, the effect of knockdown of Aurora-B on the proliferation of A549 cells and apoptosis in vitro and in vivo was investigated. The present findings indicated that silencing of Aurora-B inhibits A549 cell growth and that Aurora-B may serve as a promising therapeutic target in NSCLC.

\section{Materials and methods}

Patients and specimens. A total of 174 NSCLC and 174 adjacent tissue samples were obtained from patients that underwent surgery at The First Hospital Affiliated to Nanchang University (Nanchang, Jiangxi, China). The assessment of lymph node metastasis was performed by post-operative histological diagnosis. The histological diagnosis was determined by hematoxylin and eosin staining, according to the World Health Organization criteria (10). None of the patients had previously been administered with anti-tumor drugs or radiotherapy. Written informed consent was obtained from all subjects, and the present protocol was approved by the Institute Ethics Committee of The First Hospital Affiliated to Nanchang University.

Immunohistochemical analysis. The histological tissue sections were sliced into $4-\mu \mathrm{m}$ sections, which were subsequently stained with hematoxylin and eosin. Immunohistochemical analysis was then performed using a streptavidin-peroxidase procedure. Briefly, antigen retrieval was performed by heating 
the deparaffinized rehydrated sections in $10 \mathrm{mM}$ citrate buffer (pH 6.0; $250 \mathrm{ml}$ ) for $20 \mathrm{~min}$, followed by blocking with $10 \%$ goat serum (Gibco Life Technologies, Carlsbad, CA, USA). The sections were then incubated overnight at $4^{\circ} \mathrm{C}$ with the primary rabbit anti-human Aurora-B monoclonal antibody (cat no. ab45145; Abcam, Cambridge, UK) at a final dilution of 1:500. For the negative control, the tissue sections were incubated with phosphate-buffered saline (PBS) instead of antibodies. Subsequent to washing three times with PBS, the sections were incubated at room temperature with biotinylated secondary antibody (dilution, 1:1,000) for $40 \mathrm{~min}$, followed by incubation with horseradish peroxidase (HRP)-conjugated streptavidin for $30 \mathrm{~min}$. The sections were then stained using chemiluminescence and counterstained using hematoxylin. The stained sections were evaluated and scored by two pathological doctors in a blind manner, without prior knowledge of the clinicopathological features of the patients. The staining intensity of $\geq 500$ cells in five representative areas was determined by examination of the specimens. The expression level of Aurora-B was assessed and the intensity scores were recorded as follows: 0, no staining; 1, weak staining; 2 , moderate staining; and 3 , intense staining. The percentage scores were recorded according to the percentage of tumor cells with positive expression of Aurora-B, as follows: $0,0 \%$; $1,<10 \% ; 2,47-50 \% ; 3,51-80 \%$; and 4, 81-100\%. The final score was calculated from the average of the scores provided by the two pathological doctors, which were calculated by adding the intensity score to the percentage score. A final score $<4$ was defined as no expression, $\geq 4$ to $<5$ was defined as weak expression, $\geq 5$ to $<6$ was defined as moderate expression and $\geq 6$ was defined as strong expression.

Recombinant lentivirus vector construction. Short hairpin (sh)RNA sequences that targeted Aurora-B mRNA were designed online and manufactured by Invitrogen (Carlsbad, CA, USA), as follows: Lv-shRNA-AURKB, 5'-AGAGCTGCA CATTTGACGA-3'; and Lv-shRNA-CON, 5'-TTCTCCGAA CGTGTCACGT-3'. Lv-shRNA-CON was used as a control. The recombinant lentivirus expression plasmids were constructed, according to the manufacturer's instructions, to generate the AURKB-shRNA lentivirus (Lv-shAURKB) or negative control lentivirus (Lv-negative).

Cell culture and transfection. The human NSCLC A549 cell line was purchased from American Type Culture Collection (Manassas, VA, USA), and routinely cultured in RPMI-1640 (HyClone, Logan, UT, USA) supplemented with $10 \%$ fetal bovine serum (FBS; Sigma-Aldrich, St. Louis, MO, USA) in a humidified $37^{\circ} \mathrm{C}$ incubator containing a $5 \%$ $\mathrm{CO}_{2}$ atmosphere. The A549 cells were grown to $30-40 \%$ confluency and infected with Lv-shAURKB or Lv-negative at multiplicity of infection (MOI) of 10. To determine the infection efficiency, the cells expressing green fluorescent protein were observed using fluorescence microscopy (ECLIPSE-TS-100; Nikon, Tokyo, Japan).

Reverse transcription-quantitative polymerase chain reaction (RT-qPCR). Total RNA was isolated $48 \mathrm{~h}$ subsequent to extraction with TRIzol reagent (Invitrogen). RT-qPCR (Applied Biosystems Life Technologies, Foster City, CA, USA) was performed according to the manufacturer's instructions. The primer sequences used to amplify the cDNA compound by reverse transcriptase were as follows: Aurora-B sense, 5'-AGA AGGAGAACTCCTACC CCT-3' and antisense, 5'-CGCGTT AAGATGTCGGGTG-3'; GAPDH sense, 5'-GTGGACATC CGCAAAGAC-3' and antisense, 5'-GAAAGGGTGTAACGC AACT-3'. Six independent experiments were performed over multiple days.

Western blotting. Total protein from A549 cells was extracted using RIPA lysis buffer containing $60 \mu \mathrm{g} / \mathrm{ml}$ phenylmethylsulfonyl fluoride (Vazyme Biotech Co., Ltd., Nanjing, China). Protein concentration was determined by the Bradford assay. Western blot analysis was conducted using antibodies against Aurora-B (dilution, 1:500; Abcam) or mouse anti-human monoclonal $\beta$-actin (dilution, 1:2,000; cat no. ab8226; Cell Signaling Technology, Inc., Danvers, MA, USA). The immune complexes were detected using a pro-light HRP kit (Pierce Biotechnology, Inc., Rockford, IL, USA). All experiments were repeated six times.

Cell growth assay. Subsequent to infection with Lv-shAURKB or Lv-negative, the A549 cells were cultured in 96-well tissue culture plates at cell density of 5,000 cells per well, in RPMI-1640 containing 10\% FBS and $2 \mathrm{mM}$ glutamine. Viable proliferating cells were detected using an MTT assay. The cell viability was expressed as the optical density (OD), which was detected by an ELISA reader (Thermo MK3; Thermo Fisher Scientific, Waltham, MA, USA) at a wavelength of $490 \mathrm{~nm}$. Six independent experiments were performed over multiple days.

Apoptosis assays. A terminal deoxynucleotidyl transferase-mediated dUTP-biotin nick end labeling (TUNEL) assay was used to evaluate apoptotic activity by DNA breakage. The cells of each group were smeared on slides and fixed with $4 \%$ paraformaldehyde at room temperature for $30 \mathrm{~min}$ and then preprocessed with freshly prepared permeabilization solution containing $0.1 \%$ Triton X-100 and $0.1 \%$ sodium citrate for $15 \mathrm{~min}$ for optimal proteolysis. Apoptosis was detected using an apoptosis detection kit (Nanjing KeyGen Biotech, Co., Ltd., Nanjing, Jiangsu, China). The TdT reaction was performed for $1 \mathrm{~h}$ at $37^{\circ} \mathrm{C}$ in a humidified chamber, and sections were stained with DAPI for nuclei. TUNEL-positive cells were counted in a random selection of 10 fields and expressed as a percentage of normal nuclei under confocal laser microscopy. The overall rate of apoptosis in five fields of view was calculated using the following formula:

$$
\text { Rate of apoptosis }(\%)=\frac{\text { number of apoptotic cells }}{\text { total number of cells }} \times 100
$$

Six independent experiments were performed.

Nude mice assay. SPF grade male BALB/C nude mice, aged between 3 and 4 weeks, were obtained from the Shanghai Institute of Materia Medica (Shanghai, China), housed in sterile cages under laminar airflow hoods in a specific pathogen-free room with a 12-h light and 12-h dark schedule and fed autoclaved chow and water ad libitum. All animal procedures and 
Table I. The clinical and pathological parameters.

\begin{tabular}{|c|c|c|c|c|c|}
\hline \multirow[b]{2}{*}{ Characteristic } & \multicolumn{4}{|c|}{ Aurora-B expression } & \multirow[b]{2}{*}{ P-value } \\
\hline & - & + & ++ & +++ & \\
\hline Non-tumor tissue & 117 & 19 & 21 & 17 & 0.000 \\
\hline Lung cancer tissue & 66 & 28 & 36 & 44 & \\
\hline Age & & & & & 0.998 \\
\hline$\geq 65$ years & 36 & 15 & 19 & 24 & \\
\hline$<65$ years & 30 & 13 & 17 & 20 & \\
\hline Gender & & & & & 0.827 \\
\hline Male & 38 & 15 & 20 & 28 & \\
\hline Female & 28 & 13 & 16 & 16 & \\
\hline Tumor size & & & & & 0.000 \\
\hline$\leq 3 \mathrm{~cm}$ & 36 & 20 & 13 & 10 & \\
\hline$>3 \mathrm{~cm}$ & 30 & 8 & 23 & 34 & \\
\hline Lymph node metastasis & & & & & 0.000 \\
\hline No & 48 & 9 & 15 & 19 & \\
\hline $\mathrm{N} 1 / \mathrm{N} 2$ & 18 & 19 & 21 & 25 & \\
\hline Pathological stage & & & & & 0.001 \\
\hline $\mathrm{I} / \mathrm{II}$ & 45 & 11 & 11 & 17 & \\
\hline III/IV & 21 & 17 & 25 & 27 & \\
\hline
\end{tabular}

-, no expression; +, weak expression; ++, moderate expression; +++, strong expression.

maintenance were conducted in accordance with the institutional guidelines of the University of Nanchang (Nanchang, Jiangxi, China). The mice were injected with $0.2 \mathrm{ml}$ suspension containing $2 \times 10^{6}$ A549 cells every week until a subcutaneous tumor was formed. A suspension containing $2 \times 10^{7}$ viral particles was then injected into the formed subcutaneous tumor every other day for a week (four doses in total). The mice were sacrificed 28 days subsequent to the end of administration of viral particles and the tumors were excised. The weight of the tumors was measured.

Statistical analysis. All data were expressed as the mean \pm standard deviation. A two independent samples $t$-test was performed for statistical analysis, and the two independent samples test was used to analyze the association of the Aurora-B protein expression levels with lymph node metastasis. The association between the expression of the Aurora-B protein and the clinicopathological parameters was measured using the $\chi^{2}$ test. $\mathrm{P}<0.05$ was considered to indicate a statistically significant difference. All analyses were performed using SPSS version 13.0 (SPSS, Inc., Chicago, IL, USA).

\section{Results}

Association between Aurora-B protein expression levels in NSCLC tissues with clinicopathological parameters. Aurora-B was found to be expressed in the nucleus (Fig. 1A-D). The association identified between Aurora-B expression in NSCLC and the clinicopathological parameters is summarized in Table I. The expression levels of Aurora-B protein in NSCLC tissues was significantly increased compared with non-tumor tissues
$(\mathrm{P}=0.001)$. Notably, the positive expression rate of Aurora-B in the cases with lymph node metastasis was $78.3 \%(65 / 83)$, but only $47.3 \%$ (43/91) in those without metastasis, and this difference was significant $(\mathrm{P}<0.0001)$. The association between Aurora-B expression levels and tumor size and pathological stage was also investigated. The data revealed that Aurora-B expression was associated with the tumor size $(\mathrm{P}=0.000)$ and pathological stage $(\mathrm{P}=0.001)$. No significant association was observed between Aurora-B expression levels and other clinicopathological variables, including age at diagnosis $(\mathrm{P}=0.998)$ and gender $(\mathrm{P}=0.827)$. The present findings indicated that Aurora- $\mathrm{B}$ may contribute to the malignant phenotype in NSCLC.

Plasmid targeting Aurora-B inhibits Aurora-B expression in A549 cells. In order to investigate the effect of the inhibition of Aurora-B on the growth of A549 cells, the plasmid targeting Aurora-B was used to suppress Aurora-B expression in A549 cells. RT-PCR and western blot analysis revealed that the mRNA and protein expression of the Aurora-B were significantly decreased in cells transfected with Lv-shAURKB compared with those transfected with Lv-negative (Fig. 1E and F).

Knockdown of Aurora-B induces apoptosis and inhibits proliferation in $A 549$ cells in vitro. To explore the association between Aurora-B expression and A549 cell proliferation, cell proliferation was measured by MTT assays. The A549 cells were treated with Lv-shAURKB at a MOI of 10 . The results revealed that silencing Aurora-B led to a significant decrease in cell proliferation (Fig. 2A). Furthermore, treatment with LV-shAURKB increased the percentage of apoptotic cells, 
A

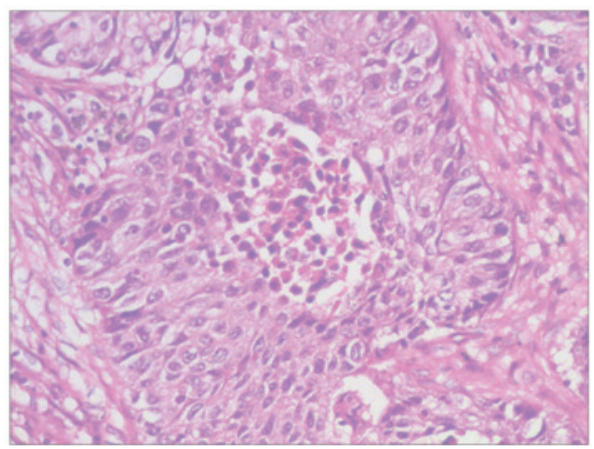

C

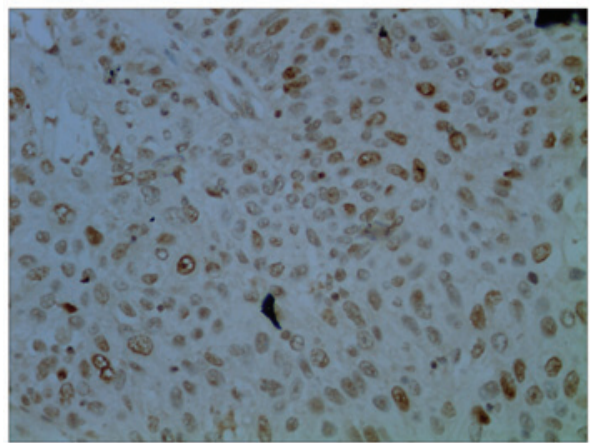

E

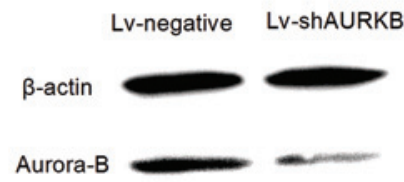

B

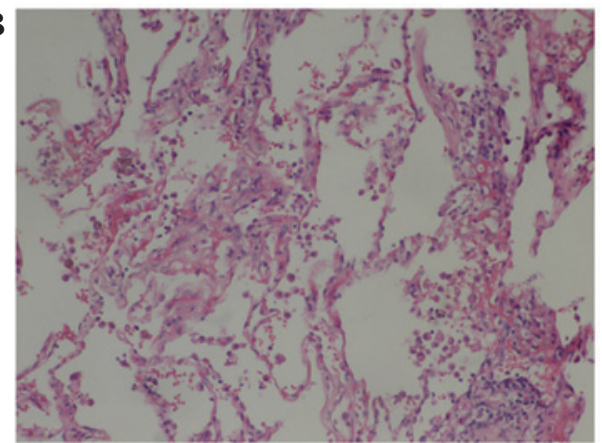

D

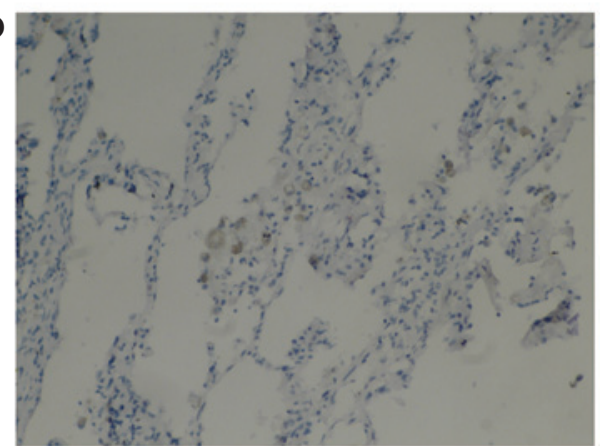

F

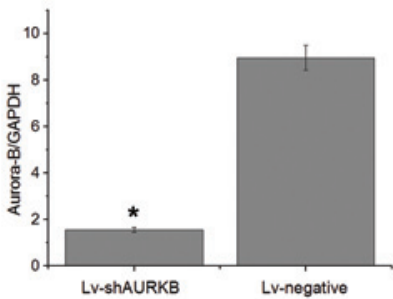

Figure 1. (A and B) Hematoxylin and eosin and (C and D) immunohistochemical staining for Aurora-B expression in (A and C) non-small cell lung cancer and (B and D) non-tumor tissues. Aurora-B was expressed in the nucleus, with significantly lower (E) protein and (F) mRNA expression in cells transfected with the Lv-shAURKB plasmid compared to Lv-negative-transfected cells. Results are expressed as the mean \pm standard deviation. " $\mathrm{P}<0.05$ vs. Lv-negative transfected group.

A

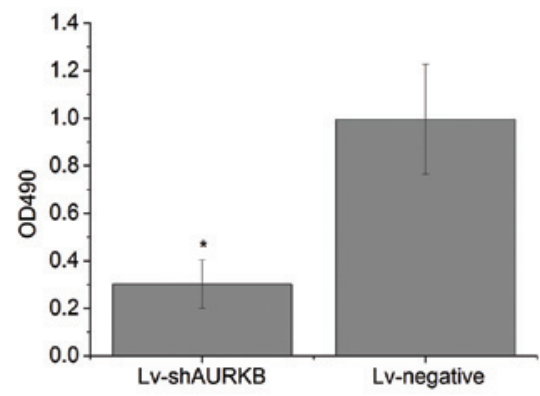

B

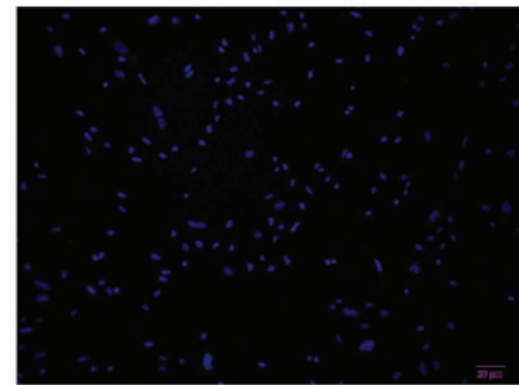

LV-ShAURKB

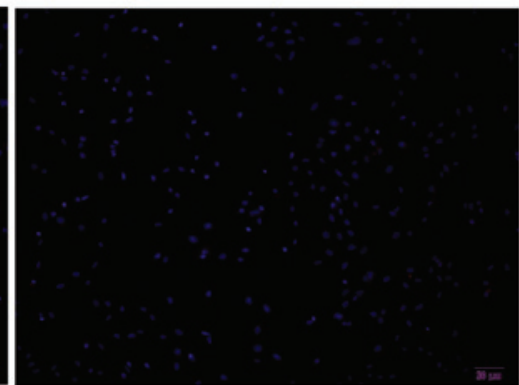

Lv-negative

Figure 2. MTT and terminal deoxynucleotidyl transferase-mediated dUTP-biotin nick end labeling assays were performed to evaluate the (A) cell proliferation and (B) apoptosis, respectively, in vitro. The results indicated that silencing Aurora-B efficiently induces cell apoptosis and inhibits cell proliferation in vitro. OD, optical density. 
A

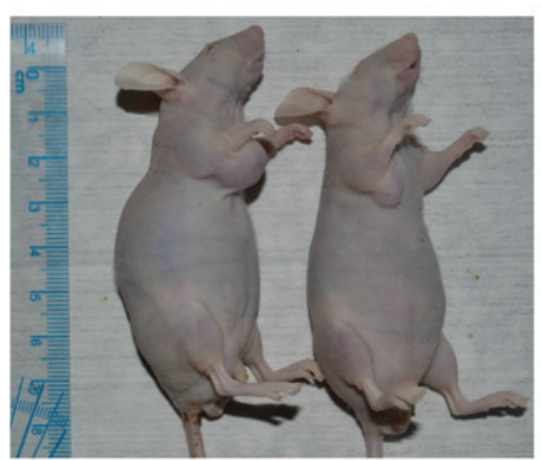

C

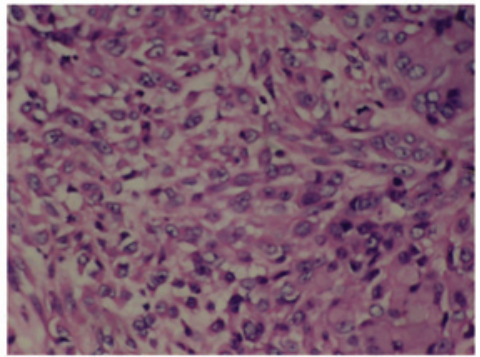

$\mathbf{E}$

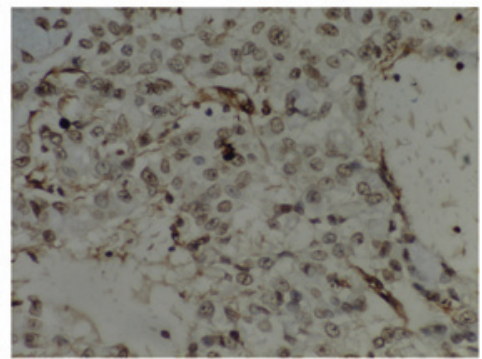

B

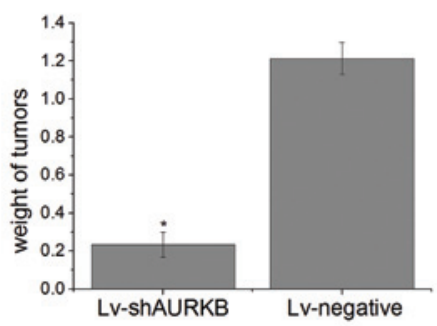

D

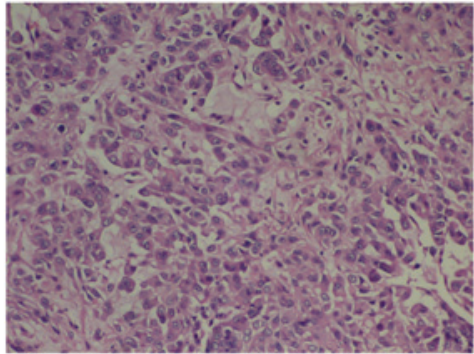

F

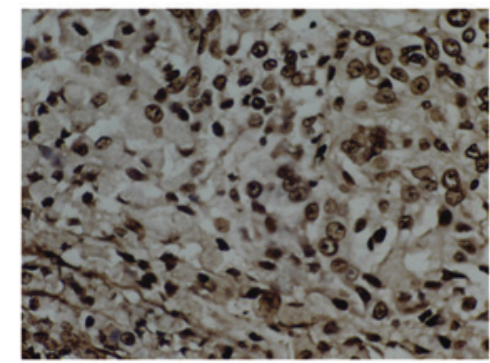

Figure 3. (A) Representative image of mice injected with Lv-shAURKB or Lv-negative viral particles. (B) Tumor growth in the mice was evaluated by measuring the weight of the tumors. The results revealed that the weight of tumors in the Lv-shAURKB-injected group was significantly lower compared with the tumor weight in the Lv-negative-injected group. (C and D) Hematoxylin and eosin staining and (E and F) terminal deoxynucleotidyl transferase-mediated dUTP-biotin nick end labeling assays were performed to evaluate the cell apoptosis in tumor tissues injected with (D and F) Lv-shAURKB or (C and E) Lv-negative viral particles. The results revealed that the apoptotic rate in the Lv-shAURKB-injected group was significantly higher compared with the apoptotic rate of the Lv-negative injected group.

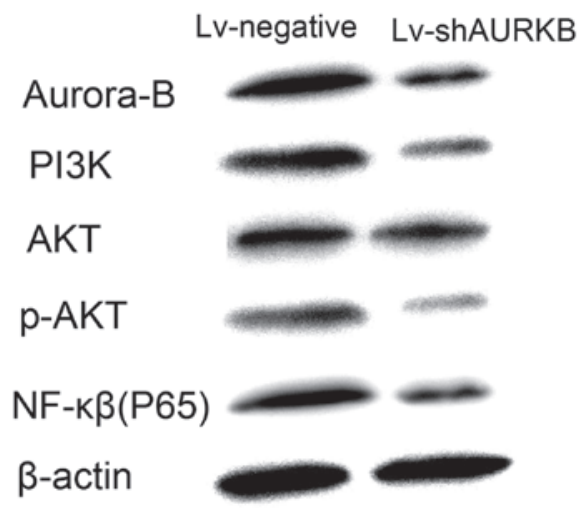

Figure 4. Western blot analysis was performed to evaluate the expression of the PI3K/AKT signaling pathway. The results revealed that PI3K, p-AKT and NF- $\mathrm{kB}(\mathrm{p} 65)$ protein expression in cells transfected with Lv-shAURKB was significantly lower compared with cells treated with Lv-negative. PI3K, phosphoinositide 3-kinase; p-AKT, phosphorylated AKT; NF-kB; nuclear factor $\kappa \mathrm{B}$.

which was determined by TUNEL (Fig. 2B). These results indicated that silencing Aurora-B may efficiently induce cell apoptosis and inhibit cell proliferation in vitro.
Knockdown of Aurora-B induces apoptosis and inhibits growth in A549 cells in vivo. To evaluate the effect of the inhibition of Aurora-B on the growth of A549 cells in vivo, a subcutaneous tumor was constructed in mice by injecting the mice with a suspension containing A549 cells. The Lv-shAURKB or Lv-negative viral particles were injected into the formed subcutaneous tumor on alternate days for a week (four doses in total). Tumor growth was evaluated by measuring the weight of the tumors. The average tumor weight in the Lv-shAURKB group was significantly decreased compared with the tumor weight in the Lv-negative group $(\mathrm{P}<0.05)$, suggesting that knockdown of Aurora-B significantly inhibited the growth of A549 cells in vivo (Fig. $3 \mathrm{~A}$ and $\mathrm{B}$ ). Furthermore, to investigate the mechanism of silencing Aurora-B on the inhibition of A549 cell growth, a TUNEL assay was performed to evaluate the cell apoptosis in tumor tissues that were obtained from mice. The results revealed that the apoptotic rate in the Lv-shAURKB group was significantly increased compared with the apoptotic rate in the Lv-negative group (Fig. 3C-F), which indicated that silencing Aurora-B may induce apoptosis in A549 cells in vivo.

Silencing Aurora-B decreases the activity of the phosphoinositide 3-kinase (PI3K)/AKT/nuclear factor (NF)- $\kappa B$ 
signaling pathway in A549 cells. In order to investigate the effect of inhibiting Aurora-B on the activity of the PI3K/AKT signaling pathway in A549 cells, the A549 cells were transfected with Lv-shAURKB or Lv-negative for $6 \mathrm{~h}$, and then cultured for $48 \mathrm{~h}$. The protein expression of PI3K, AKT, NF- $\mathrm{B}$ (p65) and phosphorylated AKT (p-AKT) was measured using western blot analysis in the A549 cells. The results revealed that the protein expression of PI3K, p-AKT and $\mathrm{NF}-\kappa \mathrm{B}(\mathrm{p} 65)$ in cells transfected with LV-shAURKB was significantly decreased compared with the expression in the cells treated with Lv-negative (Fig. 4). This indicated that Aurora-B inhibition may decrease the activity of the $\mathrm{PI} 3 \mathrm{~K} / \mathrm{AKT} / \mathrm{NF}-\kappa \mathrm{B}$ signaling pathway in A549 cells.

\section{Discussion}

Targeting the progression of mitosis is a highly successful strategy for anticancer treatment (11). Aurora kinases are serine/threonine kinases that are essential for cell cycle control and mitosis. Mammals possess three Aurora kinase family members, consisting of Aurora-A, $-\mathrm{B}$ and $-\mathrm{C}$, and these kinases are expressed at maximum levels during mitosis. Aurora-B is a component of the chromosome passenger complex (CPC), and the gene is located on the chromosome arms during prophase and at the centromeres during prometaphase and metaphase. Aurora-B has been reported to be overexpressed in numerous types of cancer (5-8).

In the present study, the expression levels of Aurora-B protein in NSCLC tissues and adjacent non-tumor tissues was detected by IHC, which revealed that the Aurora-B protein was expressed in the nucleus and the positive expression levels was significant increased in NSCLC tissues compared with the adjacent non-tumor tissues. The association between Aurora-B expression levels and clinicopathological parameters was investigated, and no association between Aurora-B expression levels and clinicopathological parameters $(\mathrm{P}>0.05)$, including gender and age at diagnosis, was observed. The NSCLC tissues used in the present study were obtained in the previous six months. To the best of our knowledge, the association between Aurora-B expression levels in NSCLC tissues and the prognosis of patients, including survival time, has not been analyzed previously. However, Kaste et al (12) and Ozger et al (13) reported that the presence of metastasis and tumor size at diagnosis are important prognosis factors for patients with osteosarcoma. Osteosarcoma and NSCLC are both malignant tumors; therefore, the presence of metastasis and tumor size at diagnosis may also be important prognostic factors for patients with NSCLC. In the present study, the expression of the Aurora-B protein in NSCLC tissues was found to be associated with the tumor size, pathological tumor stage and presence of lymph node metastasis in patients with NSCLC. The current results are similar to those obtained by Takeshita et al (14) and Wang et al (15), which indicated that Aurora-B may act as an oncogene and contribute to the malignant potential of NSCLC.

Various studies have revealed that the inhibition of Aurora-B may block cell proliferation and induce cell apoptosis in various tumor types (16-18). To evaluate the effect of the inhibition of Aurora-B on NSCLC cell proliferation and apoptosis in vitro, the plasmid targeting Aurora-B (Lv-shAURKB) was used to inhibit Aurora-B expression in A549 cells. The cell viability and apoptosis assays revealed that silencing Aurora-B induces cell apoptosis and inhibits cell proliferation in A549 cells. In addition, the association between Aurora-B expression levels and cell growth in NSCLC was investigated in vivo, and the data revealed that elevated Aurora-B expression suppresses cell apoptosis and promotes cell growth in A549 cells.

The potential molecular mechanisms associated with Aurora-B-induced apoptosis and inhibition of cell growth were also analyzed. The oncogene AKT is involved in the regulation of cell survival, and the PI3K/AKT signaling pathway regulates numerous normal cellular processes, including cell proliferation, survival, growth and motility. The aberrant activation of this pathway has been considered to be critical for tumorigenesis $(19,20)$. Long et al demonstrated that ZM447439, a small molecular inhibitor of Aurora-B, induces cell apoptosis and inhibits cell proliferation by decreasing AKT phosphorylation at Ser473 in Hep2 cancer cells (21). Accumulated data has revealed that the PI3K/AKT signaling pathway plays a key role in NSCLC malignant behavior (22-24). Therefore, it was hypothesized that silencing Aurora-B may decrease the activity of the PI3K/AKT signaling pathway in NSCLC cells. This hypothesis was confirmed in vitro and in vivo in the present study. In the current study, the protein expression of PI3K, AKT and p-AKT in A549 cells and xenograft tumors was detected by western blot analysis. The results revealed that the protein expression of PI3K and p-AKT was significantly decreased in cells and xenograft tumors treated with Lv-shAURKB compared with the expression in cells treated with Lv-negative. This finding indicated that silencing Aurora-B inhibits the activity of the PI3K/AKT signaling pathway in NSCLC tissues.

In conclusion, the present findings indicate that the knockdown of Aurora-B induces cell apoptosis and suppresses cell growth, in addition to inhibiting the activity of PI3K/AKT in NSCLC tissues. The current study indicates that targeting Aurora-B may be a potential treatment strategy for treating NSCLC.

\section{Acknowledgements}

The present study was supported by grants from the National Natural Science Foundation of China (grant no., 81260400), the Natural Science Foundation of Jiangxi Province (grant no., 20114BAB205093) and Jiangxi Province Education Department of Science and Technology (grant no., GJJ12097).

\section{References}

1. Shi L, Tang J, Tong L and Liu Z: Risk of interstitial lung disease with gefitinib and erlotinib in advanced non-small cell lung cancer: A systematic review and meta-analysis of clinical trials. Lung Cancer 83: 231-239, 2014

2. Chinn DC, Holland WS and Mack PC: Anticancer activity of the Aurora A kinase inhibitor MK-5108 in non-small-cell lung cancer (NSCLC) in vitro as monotherapy and in combination with chemotherapies. J Cancer Res Clin Oncol 140: 1137-1149, 2014.

3. Sparta AM, Bressanin D, Chiarini F, Lonetti A, Cappellini A, Evangelisti $\mathrm{C}$, et al: Therapeutic targeting of polo-like kinase-1 and Aurora kinases in T-cell acute lymphoblastic leukemia. Cell Cycle 13, 2237-2247, 2014.

4. Umene K, Banno K, Kisu I, Yanokura M, Nogami Y, Tsuji K, et al: Aurora kinase inhibitors: Potential molecular-targeted drugs for gynecologic malignant tumors. Biomed Rep 1: 335-340, 2013. 
5. Kurai M, Shiozawa T, Shih HC, Miyamoto T, Feng YZ, Kashima $\mathrm{H}$, et al: Expression of Aurora kinases A and B in normal, hyperplastic and malignant human endometrium: Aurora $\mathrm{B}$ as a predictor for poor prognosis in endometrial carcinoma. Hum Pathol 36: 1281-1288, 2005.

6. Sorrentino R, Libertini S, Pallante PL, Troncone G, Palombini L, Bavetsias V, et al: Aurora B overexpression associates with the thyroid carcinoma undifferentiated phenotype and is required for thyroid carcinoma cell proliferation. J Clin Endocrinol Metab 90: 28-35, 2005.

7. Chieffi P, Cozzolino L, Kisslinger A, Libertini S, Staibano S, Mansueto G, et al: Aurora B expression directly correlates with prostate cancer malignancy and influence prostate cell proliferation. Prostate 66: 326-333, 2006.

8. Erpolat OP, Gocun PU, Akmansu M, Karakus E and Akyol G: High expression of nuclear survivin and Aurora B predicts poor overall survival in patients with head and neck squamous cell cancer. Strahlenther Onkol 188: 248-254, 2012.

9. Li Y,Zhou W, Wei L, Jin J, Tang K, Li C, et al: The effect of Aurora kinases on cell proliferation, cell cycle regulation and metastasis in renal cell carcinoma. Int J Oncol 41: 2139-2149, 2012.

10. Minna JD, Fong K, Zochbauer-Muller S and Gazdar AF Molecular pathogenesis of lung cancer and potential translational applications. Cancer J 8: 41-46, 2002.

11. Jackson JR, Patrick DR, Dar MM and Huang PS: Targeted anti-mitotic therapies: can we improve on tubulin agents? Nat Rev Cancer 7: 107-117, 2007.

12. Kaste SC, Liu T, Billups CA, Daw NC, Pratt CB and Meyer WH: Tumor size as a predictor of outcome in pediatric non-metastatic osteosarcoma of the extremity. Pediatr Blood Cancer 43: 723-728, 2004.

13. Ozger H, Eralp L, Atalar AC, Toker B, Ayan I, Kebudi R, et al: Survival analysis and the effects of prognostic factors in patients treated for osteosarcoma. Acta Orthop Traumatol Turc 41: 211-219, 2007.

14. Takeshita M, Koga T, Takayama K, Ijichi K, Yano T, Maehara Y, et al: Aurora-B overexpression is correlated with aneuploidy and poor prognosis in non-small cell lung cancer. Lung Cancer 80: 85-90, 2013.
15. Wang WR, Yang SS, Lin JX, Zeng ZY, Liu DM and Liu HT: Expression of Aurora-B in non-small cell lung cancer and its clinical significance. Nan Fang Yi Ke Da Xue Xue Bao 29: 1853-1856, 2009 (In Chinese).

16. Ma YX and Li XZ: Effect of aurora kinase B inhibitor AZD1152 in the treatment of cisplatin-resistant ovarian carcinoma. Zhonghua Fu Chan Ke Za Zhi 48: 46-50, 2013 (In Chinese).

17. Sak A, Stuschke M, Groneberg M, Kubler D, Pottgen C and Eberhardt WE: Inhibiting the aurora B kinase potently suppresses repopulation during fractionated irradiation of human lung cancer cell lines. Int J Radiat Oncol Biol Phys 84: 492-499, 2012

18. Zhu XP, Liu ZL, Peng AF, Zhou YF, Long XH, Luo QF, et al: Inhibition of Aurora-B suppresses osteosarcoma cell migration and invasion. Exp Ther Med 7: 560-564, 2014.

19. Osaki M, Oshimura M and Ito H: PI3K-Akt pathway: its functions and alterations in human cancer. Apoptosis 9: 667-676, 2004

20. Tanno S, Yanagawa N, Habiro A, Koizumi K, Nakano Y, Osanai M, et al: Serine/threonine kinase AKT is frequently activated in human bile duct cancer and is associated with increased radioresistance. Cancer Res 64: 3486-3490, 2004

21. Long ZJ, Xu J, Yan M, Zhang JG, Guan Z, Xu DZ, et al: ZM 447439 inhibition of aurora kinase induces Hep2 cancer cell apoptosis in three-dimensional culture. Cell Cycle 7: 1473-1479, 2008.

22. Fumarola C, Bonelli MA, Petronini PG and Alfieri RR: Targeting PI3K/AKT/mTOR pathway in non small cell lung cancer. Biochem Pharmacol 90: 197-207, 2014.

23. Ma Y, Xia H, Liu Y and Li M: Silencing miR-21 sensitizes non-small cell lung cancer A549 cells to ionizing radiation through inhibition of PI3K/Akt. Biomed Res Int 2014: 617868, 2014.

24. Li YC, He SM, He ZX, Li M, Yang Y, Pang JX, et al: Plumbagin induces apoptotic and autophagic cell death through inhibition of the PI3K/Akt/mTOR pathway in human non-small cell lung cancer cells. Cancer Lett 344: 239-259, 2014. 